\title{
Mesenteric Lymphangioma with Small Intestinal Volvulus in Teenager
}

\author{
Dr. Sagar Bhalerao, Dr. Rajnish Nagarkar, Dr. Aditya Adhav, Dr. Sucheta Gandhe
}

\begin{abstract}
Lymphangioma is a benign disease characterized by roliferation of lymphatic tissue that is initiated from early failure of communication of lymphatic spaces along with normal main draining lymphatic vessels. Lymphangioma is known as a congenital malformation instead of an acquired tumor. Lymphangiomas often extend deep into the tissues while they are histologically benign. Based on current evidence, lymphangiomas are rarely observed in the mediastinum. Less than $1 \%$ of cases are observed in the retroperitoneum and mesentery region. We report an uncommon case of mesenteric lymphangioma with small intestinal volvulus in a 16-year old female. The patient underwent exploratory laparotomy followed by histological examination. As per the reports, cystic non-communicating channels of variable sizes. The patient is currently doing well and undergoing nutritional and psychological support.
\end{abstract}

\section{Index Terms - Lymphangioma, mesentery, histopathology}

\section{INTRODUCTION}

Lymphangioma can be defined as benign proliferation of lymphatic tissue that is initiated from early failure of communication of lymphatic spaces along with normal main draining lymphatic vessels. Thus, lymphangioma is considered as a congenital malformation instead of an acquired tumor [1].

Lymphangiomas are benign malformations that are rare and occur on mucous membranes or the skin. Lymphangiomas are malformations of the lymphatic system and are namely categorized as superficial or deep based on the size and depth of the abnormal lymphatic system. Lymphangiomas are often congenital in nature [2].

Cystic lymphangiomas are commonly observed in infants and young children, wherein they develop in the neck or axillary regions [3]. Histologically lymphangiomas are benign but often extends deep within the tissue [3].

As per literature, lymphangiomas rarely occur in the mediastinum while less than $1 \%$ of cases occur in the retroperitoneum and mesentery region [4].

Dr. Sagar Bhalerao, Department of medical oncology, HCG Manavata Cancer Centre

Dr. Raj Nagarkar Department of surgical oncology, HCG Manavata Cancer Centre

Dr. Aditya Adhav, Department of surgical oncology, HCG Manavata Cancer Centre

Dr. Sucheta Gandhe, Department ofpathology, HCG Manavata Cancer Centre
Lymphangiomas in most cases are asymptomatic in nature. However, they often result in several complications such as rupture with haemorrhage, secondary infection, intestinal obstruction, or volvulus. The increase in tumour size often results in these complications [4]. Mesenteric lymphangioma in some cases are known to grow to a massive size and behave aggressively in an invasive manner. Intra-abdominal mesenteric lymphangioma is a rare tumour while small bowel mesentery is a common ( $70 \%$ of cases) of intraperitoneal sites [5]. Lymphangiomas are often difficult to diagnose while surgical resection has remained an effective option for both, diagnosis and treatment in many cases. In most cases, radiological investigations are a valuable diagnostic tool while conclusive diagnosis can be confirmed by histopathology after a complete surgical resection [6]. We report an unusual case of mesenteric lymphangioma with small intestinal volvulus in a 16-year old female.

\section{CASE PRESENTATION}

A 16-year old female patient reports to the out-patient department for abdominal pain for the past 12 months. The patient had underwent a fine-needle aspiration cytology (FNAC) which revealed benign spindle cell lesion. Clinical examination revealed pare umbilical mass with no hepatosplenomegaly or ascites.

The patient underwent an ultrasonography that revealed mixed echoic heterogeneous predominantly hyperechoic with multiple small cystic areas. Lesions were observed within the left iliac fossa and pelvis. The retroperitoneal lesion measured approximately $13.4 \mathrm{~cm}$ x $6.2 \mathrm{~cm}$.

A computed tomography (CT) scan of the abdomen and pelvis was conducted. Large soft tissue mass measuring $188 \times 148 \times 44 \mathrm{~mm}$ was observed within the small bowel mesentery. As per pathological investigation, the patient's cancer antigen (CA) 125 levels were normal (16.1).

The patient underwent exploratory laparotomy for further investigation considering her clinical symptoms. As per the intraoperative findings, no live metastasis or ascites were observed. Large growth in small bowel mesentery of jejunum and proximal ileum was observed. Multiple fluid filled cavities were also noted. Frozen sections from one of the cyst walls were sent for histopathological investigation. The frozen sections were reported as spindle cell tumour.

As per the histopathological examination, the sections from mesenteric mass were observed to have cystic non communicating channels of variable sizes. These spaces are lined by attenuated endothelium resembling that in normal lymphatics (Fig. 1). The stroma is composed of delicate 
meshwork of collagen punctuated by lymphoid aggregates. Morphologically suggestive of macrocystic lymphangioma. The patient is currently doing well and undergoing nutritional and psychological support.

\section{DISCUSSION}

The incidence of benign mesenteric lymphangiomas (intra-abdominal cysts) are uncommon and account for 1 in 100,000 to 1 in 250,000 hospital admissions. Lymphangiomas are common among children, wherein nearly $40 \%$ of cases present by age one while $80 \%$ of cases present by age five [7]. Lymphangiomas are uncommon among adults and are namely observed among children as in our case. Lymphangiomas can occur anywhere in the body but the most common site is the neck which is also refeered as cystic hygromas. In context to the abdominal region, mesentery region is a common site of occurrence. However, lymphangiomas may also occur in the mesocolon, retroperitoneum, and omentum.

The clinical aetiology of lymphangiomas is not known. Intra-abdominal lymphangiomas are clinically asymptomatic. Clinicians may assess patients based on gradual increase in abdominal girth, vague abdominal pain, or sensation of fullness as key indicators for this rare condition. In most cases, patients may complain of pain ranging from chronic abdominal pain to acute severe pain due to haemorrhage into the cyst, intestinal obstruction, or compression of surrounding structures. In severe cases, the cyst also infiltrate into surrounding viscera which may lead to organ dysfunction.

In context to diagnosis of mesenteric lymphangioma, histological findings post-surgical resection of the cyst is considered standard. Till date, there are no blood tests to confirm the diagnosis. Radiological investigations such as CT help in confirming the presence of mass which may help exclude other intra-abdominal masses. However, CT alone is insufficient to provide a definite diagnosis [7]. Our case is an uncommon presentation of benign lymphangioma in a teenager. Clinicians should consider differential diagnosis of patients who have intra-abdominal cystic masses on CT scans.

\section{ACKNOWLEDGEMENT}

We would like to thank Mr. Lyndon Fernandes for his editorial assistance.
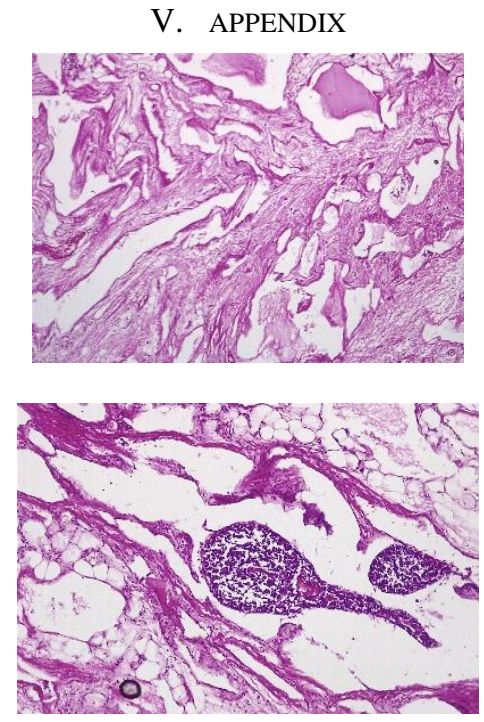

Figure 1. Histopatholgical findings: The stroma is composed of delicate meshwork of collagen punctuated by lymphoid aggregates.

\section{REFERENCES}

[1] Capasso R, Rossi E, Zeccolini M, \& Rotondo A. Lymphangioma of the small bowel mesentery in a 7-year-old girl: a report of a case investigated with ultrasonography, elastosonography and computed tomography. Journal of Ultrasound 2015;18(3), 297-300.

[2] Miceli A, Stewart KM. Lymphangioma. In: StatPearls [Internet]. Treasure Island (FL): StatPearls Publishing.

[3] Mitsukawa N, Satoh K. New treatment for cystic lymphangiomas of the face and neck: cyst wall rupture and cyst aspiration combined with sclerotherapy. J Craniofac Surg. 2012;23(4):1117-9.

[4] Watanabe, A, Suzuki, H, Kubo, N, Kobayashi, T, Araki, K, Sasaki, S et al. A case of mesenteric cystic lymphangioma in an adult which caused duodenal stenosis after resection. International Journal of Surgery Case Reports, 2013;4(2), 212-215.

[5] Tsuboi, M, Noda H, Watanabe F, Abe I, Nokubi M, \& Rikiyama T. Complete Resection of a Complicated Huge Mesenteric Lymphangioma Guided by Mesenteric Computed Tomography Angiography With Three-Dimensional Reconstruction: Report of a Case. International Surgery. 2015;100(3), 574-578.

[6] Aprea G, Guida F, Canfora A, Ferronetti A., Giugliano A, Ciciriello $\mathrm{MB}$ et al. Mesenteric cystic lymphangioma in adult: a case series and review of the literature. BMC Surgery, 2013;A4.

[7] Akwei S, Bhardwaj N, \& Murphy PD. Benign mesenteric lymphangioma presenting as acute pancreatitis: a case report. Cases Journal 2009. 2, 9328 .

First Author: Dr. Sagar Bhalerao, Department of medical oncology, HCG Manavata Cancer Centre

Has over a decade of experience in medical oncology.

Second Author: Dr. Raj Nagarkar Department of surgical oncology, HCG Manavata Cancer Centre

Is currently the managing director and chief surgeon. Has over 16 years of experience in oncology sector. Has presented in several international and national symposiums/conferences.

Third Author: Dr. Aditya Adhav, Department of surgical oncology, HCG Manavata Cancer Centre

Has over a decade of experience in surgical oncology. Has several research papers published to his credit. Has presented in several national symposiums.

Fourth Author: Dr. Sucheta Gandhe, Department ofpathology, HCG Manavata Cancer Centre

Has over a decade of experience in the field of pathology. Has several papers published to her credit. For example, Secretory Carcinoma of breast: Morphologic spectrum and importance of nuclear grade 